\title{
Shaping Greek-Tunisian Commercial Relations in the Ottoman Mediterranean World: The Efessios Merchant House ${ }^{1}$
}

\author{
Maria Christina Chatziioannou
}

\begin{abstract}
A Mediterranean Neighbourbood
Although Tunisia was never a vital economic hub, it nevertheless became part of a Greek commercial network that stretched as far east as the Black Sea. Both its Mediterranean and Ottoman identities made it attractive to Greek entrepreneurs. Moreover, its geographic proximity to western ports; its familiar socio-economic and cultural milieu; and its status as an affluent agricultural country offered a range of mercantile opportunities. Although Greece did not have strong economic ties with Tunisia in the eighteenth and nineteenth centuries, its merchants did take advantage of a widespread western network led by Jewish and French merchants. Migrants from the northwest Peloponnesus constituted a large part of the small Greek presence ( 250 persons) in Tunis in the mid-nineteenth century. ${ }^{2}$

The "Ottomanization" of Tunisia was a slow process that grew out of the Ottoman-Spanish rivalry in the Mediterranean. ${ }^{3}$ Tunisia was part of the Ottoman Empire from the sixteenth to the late nineteenth century, while in the
\end{abstract}

${ }^{1}$ An earlier version of this essay was presented at the Fourth International Congress of Maritime History in Corfu in June 2004. An alternative version of this paper appears in Maria Christina Chatziioannou and Gelina Harlaftis (eds.), Following the Nereids: Sea Routes and Maritime Business, 16th-20tth Centuries (Athens, 2006), 87-97.

${ }^{2}$ M. Kharalambis Poulos, "Les Hellenes," in C.H. Dessort, Histoire de la ville de Tunis (Algiers, 1926), 151-155 ; and H. Kazdaghli, "Communautés mediterraneennes de Tunisie. Les Grecs de Tunisie du Millet-i-rum a l'assimiliation francaise (XVIIe-XX siècles)," Revue des mondes musulmanes et de la Méditerranée, Nos. 95-98 (2002), 449-476.

${ }^{3}$ For a detailed description of the formative years of the Tunisian beylicate, see Jamil M. Abun-Nasr, "The Beylicate in Seventeenth-Century Tunisia," Intemational Joumal of Middle East Studies, VI, No. 1 (1975), 70-93.

International Journal of Maritime History, XIX, No. 1 (June 2007), 161-180. 
to independence. ${ }^{6}$ During the Ottoman occupation a local Greek elite based on trade, tax farming and piracy came into being. As well, throughout the eighteenth century French merchants became interested in agricultural exports and in the market for manufactured goods in the main Peloponnesian ports of Methoni, Coroni, Nauplio and Patras. After the French Revolution, trade with France declined throughout the eastern Mediterranean, creating openings for British economic interests and local entrepreneurs.

PeIoponnesus and Tunisia shared some important characteristics. Both were agricultural areas which exported goods to other ports in the Ottoman Empire and Western Europe. Over time, they established a complementary relationship based mainly on the maritime transport of food and raw materials.

Tunisia went through a period of internal conflict and revolt (17021814), and recent studies indicate the strengthening of bonds between local administrations and the weakening of the power of Europeans in the Regency, as well as a moderation of the corso due to peace treaties between the Porte and several European countries. Under the rule of Hammuda Pasha (17741814), the frontier culture declined in this part of North Africa, and Turkish became the official language. The whole Husaynid beylical period up to 1881 , when Tunisia became a French protectorate, has been considered as a "seminational dynasty." It was an era of the peaceful merger of Tunisian and Ottoman cultures. Driven by the idea of Islamic solidarity, the Tunisians fought alongside the Ottomans in Navarino (1827) and during the Crimean War (1854-1855), thus coming into direct conflict with Greek national aspirations.

Between 1801 and 1805, and again in 1815, the United States Navy weakened Mediterranean piracy by attacking Tunis and other corsair bases along the Barbary Coast of North Africa. ${ }^{8}$ This represented direct American intervention during a period of Anglo-French commercial competition. As a result of the loss of its revenues from piracy, the Tunisian government was plunged deeply into debt. The chief creditors were France, Italy, and Britain, all of which had imperial ambitions in northern Africa. As piracy waned, Greek merchants expanded their activities in Tunisia. But this was still not easy, since the Americans considered any vessel flying the Ottoman flag an enemy, and the Hydriots, under the Greco-Ottoman flag, were terrified at the

${ }^{6}$ Martha Pylia, "Les notables moréotes, fin du XVIII début du XIXe siècle: functions et comportments" (Unpublished $\mathrm{PhD}$ thesis, Université Paris I-Sorbonne, 2001), 203-213.

${ }^{7}$ Tunisia constituted one of the pillars of opposition towards the reform policies of Selim III; see Moalla, Regency of Tunisia, 141.

${ }^{8} \mathrm{~J}$. A. Field, Jr., America and the Mediterranean World, 1776-1882 (Princeton, 1969), 49-58. 
and cargoes. The island's main economic advantages were capital and commercial expertise, both accumulated during a long period of trade and piracy. Those advantages enabled Maltese merchants to serve as middlemen between northern European manufacturers and southern European consumers. This position enabled Malta to play an important regulatory role in the commercial activities of the western Mediterranean equivalent to that of Venice in the Adriatic. The strong Maltese presence in Tunisia is well documented, ${ }^{14}$ although Greek enterprises there still need to be explored.

The importance of Malta as a naval and military base on the Mediterranean sea routes also offered a variety of opportunities to its neighbours. Due to its strategic importance, the island also became a hub of political intrigue. It is well known, for example, that Italian political exiles who favoured the $R i$ sorgimento hatched various conspiracies and campaigns on the island.$^{15}$ Moreover, the Greek-Ottoman merchant marine frequently used Malta as a trading post. Greeks from Messolonghi and Hydra used the Maltese flag from the eighteenth century, and in the first decade of the nineteenth century cargoes of oil, cotton, wool, raisins, silk, vermilion and Cretan soap were exported from Greek ports to Malta. Indeed, the volume of Greek shipping entering Maltese ports in 1818-1820 was estimated to be almost as large as that of Greek vessels in the eastern Mediterranean during the same period. ${ }^{16}$

Between the mid-eighteenth and the early nineteenth century, Greek merchants controlled a large part of the external trade of the Ottoman Empire with Western Europe and a substantial share of intra-Ottoman commerce. Throughout this period trade in agricultural products and raw materials increased in the markets of the Ottoman Empire which were in frequent contact with ports in the Italian peninsula, France, Great Britain, the Netherlands and the Black Sea. The need for access to centres of distribution led to the creation of family enterprises whose members were dispatched to various cities. ${ }^{17}$ It is

\footnotetext{
${ }^{14}$ In 1856, the Maltese in Tunisia constituted the majority of the European population; Chater, Dépendance et mutations precoloniales, 588.

${ }^{15}$ In 1825 the Western-minded Greek politician, An. Polyzoidis, and the French philhellene, M. Raybaud, tried unsuccessfully to organise in Malta a military campaign in favour of independence; see Katerina Gardika, "O Anastasios Polizoidis kai i elliniki epanastasi," Mnemon, No. 1 (1971), 46-47.

${ }^{16}$ Vasiles Kremmydas, “Aspects des relations entre la Grèce et Malte (18101821)," in Kremmydas (ed.), Afieroma ston Niko Svorono (2 vols., Rethymno, 1986), II, 223-231.

${ }^{17}$ Maria Christina Chatzioannou, "L'emmigrazione commerciale greca dei secoli XVIII-XIX: una sfida imprenditoriale," Proposte e ricerche, XXII (1999), 22-38.
} 
Marseille and to a lesser extent Leghorn were the main destinations for Tunisian agricultural products. Oil and wool were mainly absorbed in French manufacturing. ${ }^{23}$ Marseille was also the principal destination for the small quantities of similar Peloponnesian exports. The soap factories of Marseille used oil and soda from the northwest African coast as they used olive oil from the Greek territories. It was in Marseilles and Leghorn that Greek merchants became acquainted with Tunisian products, above all the famous fezzes.

Nascent Greek mercantile enterprises had to cope with the perils of the corso at sea but still participated in the trade in Tunisian fezzes. The trade in grain carried out by the fleet from Hydra was directed mainly towards Leghorn and Marseille. These vessels not only had to cope with Barbary and Maltese corsairs but also with Greek pirates. The best-known Greek corsairs, the Maniots from the southern Peloponnesus, preyed on traffic between the Aegean and the western Mediterranean. Mani was a densely populated region which shared several demographic and economic similarities with Malta. ${ }^{24}$

The war for Greek independence was a turning point in GreekTunisian relations. ${ }^{25}$ These became openly belligerent, since the Greek fleet was engaged in a war with the Ottomans who were supported by Tunisian ships. But at the same time, the woollen Tunisian fez continued to be an important piece of Greek apparel. During the Husaynid dynasty and after the establishment of the Greek state, Greek-Tunisian trade was still conducted either by Greek mercantile networks based in Marseille and Leghorn or through nearby ports. Among the pioneers in this commerce were merchants from the southern Peloponnesus. Both regions had similar trading and consumption patterns, and their similar cultural backgrounds encouraged trade both in the Ottoman period and immediately after the achievement of Greek independence.

${ }^{23} \mathrm{Ibid}$, , 181-184.

${ }^{24} \mathrm{Mani}$ faced a continuous migration from its lands in the seventeenth through the nineteenth centuries; see V. Panayotopoulos, "I shedon nisos Mani. To geografiko ipovathro mias makras istorikis diadromis," in Y. Saitas (ed.), Mani Temoignages sur l'espace et la societe. Voyageurs et expeditions scientifiques (XVe-XIXe s.) (Athens, 1996, in Greek), 34. For Malta, see Carmel Vassallo, Corsairing to Commerce: Maltese Merchants in XVIII Century Spain (Msida, 1997), 1-3.

${ }^{25}$ Tunisian relations with the Levant became more difficult after 1822 because of the Greek war of independence and Greek corsairing activities; see M.-H. Cherif, "Expansion européenne et difficultés Tunisiennes de 1815 à 1830," Annales E.S.C., XXV, No. 3 (1970), 726. 
fathers of the family firm, Nicolettos and Stamatis, were cousins who had been partners since the mid-eighteenth century. Stamatis' line, however, was eclipsed due to a lack of male descendants, while Nicolettos' branch has survived to the present. His family members intermarried with other local notables in Calamata, such as the Benakis and Mavromihalis. ${ }^{29}$

Nicolettos's principal heir was Elias Efessios (1789-1867), an adventurous young man who at the beginning of the nineteenth century left his birthplace and went to Smyrna, where he stayed with an Armenian family. He began to sail on the dangerous seas between Calamata, Smyrna and Alexandria at a time when all three were part of the Ottoman Empire. In 1816 Elias moved to Tunis and began to trade. He initiated the creation of a commercial network by establishing correspondents in Malta and Zante; from 1823 to 1828 , the Maltese trade was handled through Nicolettos Efessios. After the creation of the Greek state, Elias moved back to Calamata; married a woman from the family of his Constantinople correspondent; and extended the family by fathering seven children. One of his daughters, Charikleia, married Petros Ant. Mavromibalis, the mayor of Calamata and a descendant of a well-known Maniot family. Elias became a successful entrepreneur who profited from family expertise and continued to accumulate wealth for many years to come.

Table 1

\begin{tabular}{|c|c|c|c|}
\hline \multicolumn{4}{|c|}{ Efessios Merchant Network Locations (1831-1861) } \\
\hline Network Suppliers & $\begin{array}{c}\text { Entrepreneur Co- } \\
\text { ordinator }\end{array}$ & $\begin{array}{l}\text { Information } \\
\text { Agents }\end{array}$ & $\begin{array}{l}\text { Network Sup- } \\
\text { pliers }\end{array}$ \\
\hline \multicolumn{4}{|l|}{ Constantinople } \\
\hline \multicolumn{4}{|l|}{ Corfu } \\
\hline \multicolumn{4}{|l|}{ Coroni } \\
\hline Hydra & & Leghorn & \\
\hline Kyparissia & Calamata & Malta & Tunis \\
\hline \multicolumn{4}{|l|}{ Leontari } \\
\hline \multicolumn{4}{|l|}{ Mistras } \\
\hline \multicolumn{4}{|l|}{ Nissi } \\
\hline \multicolumn{4}{|l|}{ Patras } \\
\hline \multicolumn{4}{|l|}{ Pyrgos } \\
\hline Zante & & & \\
\hline
\end{tabular}

Source: Efessios Archive, Commercial Correspondence, 1839-1850, and Bills of Lading, 1831-1861.

${ }^{29}$ The relations are certified by marriage contracts and documents of real estate purchases in the Efessios' private archive. 
It is clear based upon the bills of lading that the peak period of activity was 1838-1849, the decade following Elias Efessios' relocation to Calamata and the formative years of his personal trading network. In the same decade voyages between Calamata and Tunis, which constituted the majority of trading ventures, were mainly in exports from Tunis (see appendix 1) ${ }^{31}$

Over the thirty-year period the dominant export from Calamata to Tunis was silk, accompanied occasionally by vermillion. In the other direction only fifteen cases of red fezzes were shipped from Tunis to Calamata along with dried octopus, stockfish and occasionally butter, rosewater and dates. This trading pattern is reflected in the firm's business correspondence: petty commodities accompanying the most expensive trading items such as silk and fezzes. This pattern has been observed in similar case studies in periods when foreign competition was intense and medium-rank merchants could not purchase those goods in greatest demand. ${ }^{32}$ The withdrawal of the famous Tunisian fezzes from the Efessios' commercial exchanges cannot be explained by a decline in consumer demand, since fezzes continued to be worn throughout Greece until the late nineteenth century. Another explanation might have more validity: that a large part of domestic demand was satisfied by a new manufacturer who commenced production in Athens in 1839, offering an assortment of fezzes that competed with Tunisian exports. ${ }^{33}$

The export cargoes from Tunis to Calamata included 164,000 silver coins (spanish colonati, talers and francs). This amount certainly was not accounted for solely by remittances for Greek exports, so we need to seek an alternate explanation. One hypothesis worth testing is that the founding of the Greek state created a demand for European coins for domestic commercial transactions and that Tunis was an excellent locale for their acquisition.

The Efessios merchant house reflected new economic realities when it moved away from traditional trades. In the southern Peloponnesus silk had long been produced and manufactured in Calamata and its hinterland, as was vermillion. Similarly, fezzes and dried octopus from Sfax had long been staples of Mediterranean trade. But all these products were relics of an increasingly outmoded trading pattern. Navigating in dangerous seas and carrying traditional products throughout the Mediterranean basin reflected the economic needs of a different era.

\footnotetext{
${ }^{31}$ See appendix 1 .
}

${ }^{32}$ The situation was similar in Smyrna for the Geroussi family; see Maria Christina Chatziioannou, Oikogeneiaki stratigiki kai emporikos antagonismos. $O$ oikos Geroussi ston 190 aiona (Athens, 2003, in Greek).

${ }^{33}$ Christina Agriantoni, Oi aparhes tis ekviomihanisis stin Ellada ton 190 aiona (Athens, 1986), 398 (in Greek). 


\begin{tabular}{|c|c|c|c|c|c|c|}
\hline Date & $\begin{array}{l}\text { Con- } \\
\text { signor }\end{array}$ & Place & Consignee & Place & $\begin{array}{l}\text { Ship } \\
\text { (Flag) }\end{array}$ & Cargo \\
\hline 1836 & $\begin{array}{l}\text { Efessios, } \\
\text { Elias }\end{array}$ & Calamata & Gialias, P. & Tunis & Greek & $\begin{array}{l}9 \text { sacks silk, } \\
\text { figs, dresses }\end{array}$ \\
\hline 1836 & $\begin{array}{l}\text { Argyropo } \\
\text { ulos, All. }\end{array}$ & Tunis & $\begin{array}{l}\text { Efessios, } \\
\text { Elias }\end{array}$ & Calamata & Greek & $\begin{array}{l}19 \text { quintals } \\
\text { octopus }\end{array}$ \\
\hline 1836 & Gialias, P. & Tunis & $\begin{array}{l}\text { Efessios, } \\
\text { Elias }\end{array}$ & Calamata & Greek & $\begin{array}{l}2652 \text { silver } \\
\text { coins (Spanish } \\
\text { colonati, talers } \\
\text { spathati) }\end{array}$ \\
\hline 1836 & $\begin{array}{l}\text { Efessios, } \\
\text { Elias }\end{array}$ & Calamata & Gialias, $P$. & Tunisia & Greek & 26 sacks silk \\
\hline 1836 & $\begin{array}{l}\text { Tzatzonis, } \\
\mathrm{K} \text {. }\end{array}$ & Patras & $\begin{array}{l}\text { Efessios, } \\
\text { Elias }\end{array}$ & Calamata & Greek & 7 sacks silk \\
\hline 1836 & $\begin{array}{l}\text { Gialias, } \\
\text { P. }\end{array}$ & Tunis & $\begin{array}{l}\text { Efessios, } \\
\text { Elias }\end{array}$ & Calamata & Greek & $\begin{array}{l}567 \text { silver } \\
\text { coins (Spanish } \\
\text { colonati, talers } \\
\text { spathati) }\end{array}$ \\
\hline 1837 & Gialias, $P$. & Tunis & $\begin{array}{l}\text { Efessios, } \\
\text { Elias }\end{array}$ & Calamata & Greek & $\begin{array}{l}1646 \text { silver } \\
\text { coins (Spanish } \\
\text { colonati, talers } \\
\text { spathati) }\end{array}$ \\
\hline 1837 & $\begin{array}{l}\text { Efessios, } \\
\text { Elias }\end{array}$ & Calamata & Gialias, $P$. & Tunis & Greek & 22 sacks silk \\
\hline 1838 & Gialias, $P$. & Tunis & $\begin{array}{l}\text { Efessios, } \\
\text { Elias }\end{array}$ & Calamata & Greek & $\begin{array}{l}4173 \text { silver } \\
\text { coins (Spanish } \\
\text { colonati, talers } \\
\text { spathati), } 15 \\
\text { diamond rings }\end{array}$ \\
\hline 1838 & Gialias, $P$. & Tunis & $\begin{array}{l}\text { Efessios, } \\
\text { Elias }\end{array}$ & Calamata & Greek & $\begin{array}{l}19 \text { quintals } \\
\text { octopus, } 21 \\
\text { straw bags, } \\
\text { dates, tobacco }\end{array}$ \\
\hline 1838 & $\begin{array}{l}\text { Efessios, } \\
\text { Elias }\end{array}$ & Zante & $\begin{array}{l}\text { Efessios, } \\
\text { Elias }\end{array}$ & Calamata & Greek & $\begin{array}{l}\text { l case } f e z, \\
\text { treated silk, } \\
\text { cloth items }\end{array}$ \\
\hline 1838 & Gialias, $P$. & Tunis & $\begin{array}{l}\text { Efessios, } \\
\text { Elias }\end{array}$ & Calamata & Greek & $\begin{array}{l}3000 \text { silver } \\
\text { coins (talers, } \\
\text { francs) }\end{array}$ \\
\hline 1838 & Gialias, P. & Tunis & $\begin{array}{l}\text { Efessios, } \\
\text { Elias }\end{array}$ & Calamata & Greek & $\begin{array}{l}4000 \text { silver } \\
\text { coins (talers, } \\
\text { francs) }\end{array}$ \\
\hline 1838 & Gialias, P. & Tunis & $\begin{array}{l}\text { Efessios, } \\
\text { Elias }\end{array}$ & Calamata & Greek & $\begin{array}{l}634 \text { silver } \\
\text { francs of Mar- } \\
\text { seille }\end{array}$ \\
\hline
\end{tabular}




\begin{tabular}{|c|c|c|c|c|c|c|}
\hline Date & $\begin{array}{l}\text { Con- } \\
\text { signor }\end{array}$ & Place & Consignee & Place & $\begin{array}{l}\text { Ship } \\
\text { (Flag) }\end{array}$ & Cargo \\
\hline 1840 & $\begin{array}{l}\text { Giamari } \\
\text { and Bas- } \\
\text { togi }\end{array}$ & Leghorn & $\begin{array}{l}\text { Efessios, } \\
\text { Elias }\end{array}$ & Calamata & Greek & $\begin{array}{l}280 \text { bales } \\
\text { stockfish }\end{array}$ \\
\hline 1841 & $\begin{array}{l}\text { Vassallo, } \\
\text { Giuseppe }\end{array}$ & Sfax & $\begin{array}{l}\text { Efessios, } \\
\text { Elias/Scou } \\
\text { teridi, G. }\end{array}$ & $\begin{array}{l}\text { Calamata! } \\
\text { Zante }\end{array}$ & $\begin{array}{l}\text { Sar- } \\
\text { dinia }\end{array}$ & $\begin{array}{l}151 \text { quintals } \\
\text { octopus }\end{array}$ \\
\hline 1841 & $\begin{array}{l}\text { Efessios, } \\
\text { Elias }\end{array}$ & Calamata & $\begin{array}{l}\text { Argyrou- } \\
\text { lakis, An. }\end{array}$ & Tunis & Greek & 1 parcel silk \\
\hline 1841 & $\begin{array}{l}\text { Efessios, } \\
\text { Elias }\end{array}$ & Calamata & Gialias, P. & Tunis & Greek & $\begin{array}{l}7 \text { sacks silk, } 2 \\
\text { sacks vermil- } \\
\text { lion }\end{array}$ \\
\hline 1841 & $\begin{array}{l}\text { Efessios, } \\
\text { Elias }\end{array}$ & Calamata & Gialias, P. & Tunis & Greek & $\begin{array}{l}18 \text { sacks silk, } \\
1 \text { sack vermil- }\end{array}$ \\
\hline$\therefore$ & - & & & & . & $\begin{array}{l}\text { lion, } 18 \text { pieces } \\
\text { cheese }\end{array}$ \\
\hline 1841 & $\begin{array}{l}\text { Kyrousis, } \\
\text { I. }\end{array}$ & Tunis & $\begin{array}{l}\text { Efessios, } \\
\text { Elias }\end{array}$ & Calamata & Greek & 500 francs \\
\hline 1841 & Gialias, $P$. & Tunis & $\begin{array}{l}\text { Efessios, } \\
\text { Elias }\end{array}$ & Calamata & Greek & 1000 francs \\
\hline 1841 & Gialias, $P$. & Tunis & $\begin{array}{l}\text { Efessios, } \\
\text { Elias }\end{array}$ & Calamata & Greek & 1169 francs \\
\hline 1841 & Gialias, $\mathbf{P}$. & Tunis & $\begin{array}{l}\text { Efessios, } \\
\text { Elias }\end{array}$ & Calamata & Greek & 750 francs \\
\hline 1841 & Gialias, $P$. & Tunis & $\begin{array}{l}\text { Efessios, } \\
\text { Elias }\end{array}$ & Calamata & Greek & $\begin{array}{l}3026 \text { silver } \\
\text { coins (talers, } \\
\text { francs) }\end{array}$ \\
\hline 1841 & $\begin{array}{l}\text { Kyrousis, } \\
\text { I. }\end{array}$ & Tunis & $\begin{array}{l}\text { Efessios, } \\
\text { Elias }\end{array}$ & Calamata & Greek & 300 francs \\
\hline 1841 & $\begin{array}{l}\text { Ardiro- } \\
\text { poulos, } \\
\text { An. }\end{array}$ & Tunis & $\begin{array}{l}\text { Efessios, } \\
\text { Elias }\end{array}$ & Calamata & $\begin{array}{l}\text { Sar- } \\
\text { dinian }\end{array}$ & 307 ox heads \\
\hline 1841 & Gialias, P. & Tunis & $\begin{array}{l}\text { Efessios, } \\
\text { Elias }\end{array}$ & Cálamata & Greek & 860 francs \\
\hline 1841 & Gialias, $P$. & Tunis & $\begin{array}{l}\text { Efessios, } \\
\text { Elias }\end{array}$ & Calamata & $\begin{array}{l}\text { Sar- } \\
\text { dinian }\end{array}$ & 3000 francs \\
\hline 1841 & Gialias, P. & Tunis & $\begin{array}{l}\text { Efessios, } \\
\text { Elias }\end{array}$ & Calamata & $\begin{array}{l}\text { Sar- } \\
\text { dinian }\end{array}$ & 1891 francs \\
\hline 1841 & $\begin{array}{l}\text { Efessios, } \\
\text { Elias }\end{array}$ & Calamata & $\begin{array}{l}\text { Stuaritis, } \\
\text { I. }\end{array}$ & Zante & Ionian & 1 sack silk \\
\hline 1841 & $\begin{array}{l}\text { Kyrousis, } \\
\text { I. }\end{array}$ & Tunis & $\begin{array}{l}\text { Efessios, } \\
\text { Elias }\end{array}$ & Calamata & Greek & 159 francs \\
\hline 1841 & Gialias, P. & Tunis & $\begin{array}{l}\text { Efessios, } \\
\text { Elias }\end{array}$ & Calamata & Greek & $\begin{array}{l}2750 \text { silver } \\
\text { coins (talers, } \\
\text { francs) }\end{array}$ \\
\hline
\end{tabular}




\begin{tabular}{|c|c|c|c|c|c|c|}
\hline Date & $\begin{array}{l}\text { Con- } \\
\text { signor }\end{array}$ & Place & Consignee & Place & $\begin{array}{l}\text { Ship } \\
\text { (Flag) }\end{array}$ & Cargo \\
\hline 1844 & Gialias, P. & Tunis & $\begin{array}{l}\text { Efessios, } \\
\text { Elias }\end{array}$ & Calamata & $\begin{array}{l}\text { Sar- } \\
\text { dinian }\end{array}$ & 500 talers \\
\hline 1844 & Gialias, P. & Tunis & $\begin{array}{l}\text { Efessios, } \\
\text { Elias }\end{array}$ & Calamata & $\begin{array}{l}\text { Tus- } \\
\text { can }\end{array}$ & $\begin{array}{l}6000 \text { silver } \\
\text { coins (talers, } \\
\text { francs) }\end{array}$ \\
\hline 1844 & $\begin{array}{l}\text { Efessios, } \\
\text { Elias }\end{array}$ & Calamata & $\begin{array}{l}\text { Bagdante- } \\
\text { lou, G. }\end{array}$ & Zante & lonian & 978 francs \\
\hline 1844 & Gialias, P. & Tunis & $\begin{array}{l}\text { Efessios, } \\
\text { Elias }\end{array}$ & Calamata & Greek & $\begin{array}{l}27 \text { sacks silk, } \\
\text { figs }\end{array}$ \\
\hline 1845 & $\begin{array}{l}\text { Theo- } \\
\text { dorou, G. }\end{array}$ & Calamata & $\begin{array}{l}\text { Efessios, } \\
\text { Elias }\end{array}$ & Tunis & Ionian & $\begin{array}{l}9 \text { sacks silk, } \\
\text { vermillion, } \\
\text { figs }\end{array}$ \\
\hline 1845 & $\begin{array}{l}\text { Efessios, } \\
\text { Elias }\end{array}$ & Calamata & $\begin{array}{l}\text { Efessios, } \\
\text { Elias }\end{array}$ & Patras & Greek & 4 sacks silk \\
\hline 1845 & $\begin{array}{l}\text { Efessios, } \\
\text { Elias }\end{array}$ & Tunis & $\begin{array}{l}\text { Karalias, } \\
\text { I. }\end{array}$ & Calamata & $\begin{array}{l}\text { Sar- } \\
\text { dinian }\end{array}$ & 1000 talers \\
\hline 1846 & $\begin{array}{l}\text { Efessios, } \\
\text { Elias }\end{array}$ & Tunis & $\begin{array}{l}\text { Karalias, } \\
\text { I. }\end{array}$ & Calamata & Greek & $\begin{array}{l}\text { Muhamiz, } \\
\text { dates }\end{array}$ \\
\hline 1846 & $\begin{array}{l}\text { Efessios, } \\
\text { Elias }\end{array}$ & Tunis & $\begin{array}{l}\text { Karalias, } \\
\text { I. }\end{array}$ & Calamata & $\begin{array}{l}\text { Aus- } \\
\text { trian }\end{array}$ & 4000 scuds \\
\hline 1846 & $\begin{array}{l}\text { Efessios, } \\
\text { Elias }\end{array}$ & Tunis & $\begin{array}{l}\text { Karalias, } \\
\text { I. }\end{array}$ & Calamata & $\begin{array}{l}\text { Sar- } \\
\text { dinian }\end{array}$ & 1000 francs \\
\hline 1846 & $\begin{array}{l}\text { Efessios, } \\
\text { Elias }\end{array}$ & Tunis & $\begin{array}{l}\text { Efessios, } \\
\text { Elias }\end{array}$ & $\begin{array}{l}\text { Malta/ } \\
\text { Calamata }\end{array}$ & Greek & $\begin{array}{l}10 \text { cases fez, } \\
\text { garments, } \\
\text { kina, marble, } \\
\text { wool, latroni, } \\
\text { butter, caviar, } \\
1 \text { French chan- } \\
\text { delier, } 9000\end{array}$ \\
\hline & & & & & & talers \\
\hline 1847 & $\begin{array}{l}\text { Efessios, } \\
\text { Elias }\end{array}$ & Calamata & $\begin{array}{l}\text { Krassa- } \\
\text { kopoulos, } \\
\text { El. }\end{array}$ & Tunis & Greek & 8 sacks silk \\
\hline 1847 & $\begin{array}{l}\text { Krassa- } \\
\text { kopoulos, } \\
\text { El. }\end{array}$ & Tunis & $\begin{array}{l}\text { Efessios, } \\
\text { Elias }\end{array}$ & Calamata & $\begin{array}{l}\text { Sar- } \\
\text { dinian }\end{array}$ & 4000 talers \\
\hline 1847 & $\begin{array}{l}\text { Manida- } \\
\text { kis, Chr. }\end{array}$ & Tunis & $\begin{array}{l}\text { Efessios, } \\
\text { Elias }\end{array}$ & Calamata & & $\begin{array}{l}250 \text { silver } \\
\text { coins (talers, } \\
\text { francs) }\end{array}$ \\
\hline 1847 & $\begin{array}{l}\text { Krassa- } \\
\text { kopolous, } \\
\text { El. }\end{array}$ & Tunis & $\begin{array}{l}\text { Efessios, } \\
\text { Elias }\end{array}$ & Calamata & Greek & 3500 talers \\
\hline
\end{tabular}




\begin{tabular}{|c|c|c|c|c|c|c|}
\hline Date & $\begin{array}{l}\text { Con- } \\
\text { signor }\end{array}$ & Place & Consignee & Place & $\begin{array}{l}\text { Ship } \\
\text { (Flag) }\end{array}$ & Cargo \\
\hline 1851 & $\begin{array}{l}\text { Krassa- } \\
\text { kopoulos, } \\
\text { El. }\end{array}$ & Tunis & $\begin{array}{l}\text { Efessios, } \\
\text { Elias }\end{array}$ & Calamata & Greek & 2000 talers \\
\hline 1852 & $\begin{array}{l}\text { Efessios, } \\
\text { Elias }\end{array}$ & Calamata & $\begin{array}{l}\text { Krassa- } \\
\text { kopoulos, } \\
\text { El. }\end{array}$ & Tunis & Greek & $\begin{array}{l}12 \text { sacks silk, } \\
\text { vermillion, } \\
\text { figs, cheese, } \\
\text { caviar }\end{array}$ \\
\hline 1852 & $\begin{array}{l}\text { Efessios, } \\
\text { Elias }\end{array}$ & Calamata & $\begin{array}{l}\text { Krassa- } \\
\text { kopoulos, } \\
\text { El. }\end{array}$ & Tunis & $\begin{array}{l}\text { Rus- } \\
\text { sian }\end{array}$ & $\begin{array}{l}8000 \text { silver } \\
\text { coins (talers, } \\
\text { francs) }\end{array}$ \\
\hline 1852 & $\begin{array}{l}\text { Krassa- } \\
\text { kopoulos, } \\
\text { El. }\end{array}$ & Tunis & $\begin{array}{l}\text { Efessios, } \\
\text { Elias }\end{array}$ & Calamata & $\begin{array}{l}\text { Sar- } \\
\text { dinian }\end{array}$ & $\begin{array}{l}5000 \text { francs, } \\
12 \text { flasks } \\
\text { rosewater, } \\
\text { Muhamiz }\end{array}$ \\
\hline 1852 & $\begin{array}{l}\text { Efessios, } \\
\text { Elias }\end{array}$ & Calamata & $\begin{array}{l}\text { Krassa- } \\
\text { kopoulos, } \\
\text { El. }\end{array}$ & Tunis & $\begin{array}{l}\text { Rus- } \\
\text { sian }\end{array}$ & $\begin{array}{l}15 \text { sacks sillk, } \\
8 \text { sacks ver- } \\
\text { million, cav- } \\
\text { iar, cheese, } \\
\text { sausages }\end{array}$ \\
\hline 1853 & $\begin{array}{l}\text { Niculi, } \\
\text { Dionisio }\end{array}$ & Sfax & $\begin{array}{l}\text { Efessios, } \\
\text { Elias }\end{array}$ & Calamata & French & $\begin{array}{l}32 \text { sacks octo- } \\
\text { pus, } 7936 \\
\text { rotoli }\end{array}$ \\
\hline 1853 & $\begin{array}{l}\text { Krassa- } \\
\text { kopoulos, } \\
\text { El. }\end{array}$ & Tunis & $\begin{array}{l}\text { Efessios, } \\
\text { Elias }\end{array}$ & Calamata & Greek & $\begin{array}{l}7500 \text { francs, } \\
\text { rosewater }\end{array}$ \\
\hline 1853 & $\begin{array}{l}\text { Alamag- } \\
\text { kas, I. }\end{array}$ & Malta & $\begin{array}{l}\text { Efessios, } \\
\text { Elias }\end{array}$ & Calamata & Greek & $\begin{array}{l}1 \text { iron double } \\
\text { bed }\end{array}$ \\
\hline 1854 & $\begin{array}{l}\text { Krassa- } \\
\text { kopoulos, } \\
\text { El. }\end{array}$ & Tunis & $\begin{array}{l}\text { Efessios, } \\
\text { Elias }\end{array}$ & Calamata & $\begin{array}{l}\text { Sar- } \\
\text { dinian }\end{array}$ & 1 case $f e z$ \\
\hline 1854 & $\begin{array}{l}\text { Krassa- } \\
\text { kopoulos, } \\
\text { El. }\end{array}$ & Tunis & $\begin{array}{l}\text { Efessios, } \\
\text { Elias }\end{array}$ & Calamata & $\begin{array}{l}\text { Sar- } \\
\text { dinian }\end{array}$ & $\begin{array}{l}1100 \text { francs, } 1 \\
\text { case fez }\end{array}$ \\
\hline 1854 & $\begin{array}{l}\text { Efessios, } \\
\text { Elias }\end{array}$ & Calamata & $\begin{array}{l}\text { Ralli, } \\
\text { Skilitzi, } \\
\text { Argenti }\end{array}$ & Marseille & Greek & 8 sacks silk \\
\hline 1855 & $\begin{array}{l}\text { Krassa- } \\
\text { kopoulos, } \\
\text { El. }\end{array}$ & Tunis & $\begin{array}{l}\text { Efessios, } \\
\text { Elias }\end{array}$ & Calamata & $\begin{array}{l}\text { Sar- } \\
\text { dinian }\end{array}$ & 8395 francs \\
\hline 1855 & $\begin{array}{l}\text { Alamag- } \\
\text { kas, I. }\end{array}$ & Malta & $\begin{array}{l}\text { Efessios, } \\
\text { Elias }\end{array}$ & Calamata & Greek & $\begin{array}{l}47 \text { sacks octo- } \\
\text { pus }\end{array}$ \\
\hline 1856 & $\begin{array}{l}\text { Francesos, } \\
\text { I. }\end{array}$ & Syros & $\begin{array}{l}\text { Efessios, } \\
\text { Elias }\end{array}$ & Calamata & Greek & 1000 francs \\
\hline 18 & $\begin{array}{l}\text { Francesos, } \\
\text { I. }\end{array}$ & Syros & $\begin{array}{l}\text { Efessios, } \\
\text { Elias }\end{array}$ & Calamata & Greek & $\begin{array}{l}966 \text { francs, } \\
197 \text { pounds } \\
\text { Sterling }\end{array}$ \\
\hline
\end{tabular}

